\title{
SUGER ET LES ARCHIVES: EN RELISANT DEUX PASSAGES DU DE ADMINISTRATIONE
}

Nul abbé, probablement, ne fut soucieux plus que Suger de son wautopromotion«, de transmettre »à la mémoire de la postérité« les traces de son activité, de marquer "par le calame et l'encre « l'étendue de son action au service du saint et du sanctuaire auxquels il était voué ${ }^{2}$. Outre un superbe volet narratif, cette production d'écriture comporte tout un pan diplomatique formé des chartes que Suger prit soin de rédiger ou de faire rédiger, à son nom ${ }^{3}$ ou au nom du souverain régnant, ou bien encore au nom de souverains morts depuis des siècles (je veux parler naturellement des faux). La plupart de ces chartes avaient vocation à enrichir un chartrier où dialoguaient des pièces de toutes époques, de toutes origines et de toutes formes, un creuset qui tenait du trésor, du mémorial et de l'arsenal ${ }^{4}$. Les rudes combats menés par les dionysiens contre l'ordinaire ecclésiastique dans les années 1060 avaient probablement forgé une solide »culture« de l'arme archivistique ${ }^{5}$; Suger en fut peut-être

1 Cette formule très répandue est reprise par Suger en son De administratione; cf. Suger, Euvres, t. 1: Écrit sur la consécration de Saint-Denis, l'Guvre administrative, Histoire de Louis VII, éd. et trad. Françoise GASPARRI, Paris 1996 (Classiques de l'histoire de France au Moyen Âge, 37), p. 54-55.

2 Voir Françoise GASPARRI, L'abbé Suger de Saint-Denis, mémoire et perpétuation des œuvres humaines, dans: Cahiers de civilisation médiévale 44 (2001), p. 247-257.

3 Dans le sillage de son prédécesseur Albert LECOY DE LA MARCHE (Suger, Euvres complètes, Paris 1867 [Société de l'histoire de France]), Françoise GASPARRI a incorporé dans sa publication les 16 chartes actuellement connues qui soient intitulées au nom de Suger: Cuvres, t. 2: Lettres de Suger, Chartes de Suger, Vie de Suger par le moine Guillaume, Paris 2001 (Classiques de l'histoire de France au Moyen Âge, 41), p. 155-267. Sur les chartes abbatiales de Suger: EAD., Suger de Saint-Denis. Pratiques, formes, langage d'une culture écrite au XII siècle, à propos d'une charte originale jusqu'ici inconnue, Paris, Arch. nat. S 2247 n $^{\circ}$ 3, dans: Scrittura e Civiltà 20 (1996), p. 111-135.

4 Trois des seize chartes de Suger proviennent de fonds d'archives étrangers à Saint-Denis: éd. GASPARRI (voir n. 3), $\mathrm{n}^{\circ} 14$, p. 258-263 (1148-49, prieuré parisien de Saint-Martin-desChamps), ${ }^{\circ} 15$, p. $262-265$ (1149, abbaye de Valséry au dioc. de Soissons), n 16, p. 264 267 (1150, abbaye de Longpont au même dioc.). Deux autres sont issues du cartulaire du prieuré dionysien de La Chapelaude $\left(n^{\circ} 4\right.$, p. 178-181: 1133; $\mathbf{n}^{\circ}$ 7, p. 190-193: 1126-1136).

5 Sur ce conflit, voir la mise en perspective de Rolf GRosSE, Saint-Denis zwischen Adel und König. Die Zeit vor Suger (1053-1122), Stuttgart 2002 (Beihefte der Francia, 57), p. 61-70, avec référence à l'étude fondamentale de Léon LEVILLAIN sur les faux élaborés à cette occasion: Études sur l'abbaye de Saint-Denis à l'époque mérovingienne. III: »Privilegium« et 
nourri après son entrée au monastère quelque trente ans plus tard. En tout cas, il eut le sens et le goût de l'écrit diplomatique et des archives.

Comme nul ne l'ignore - on va cependant y revenir -, Suger s'est plu à rappeler dans son De administratione $e^{6}$ combien dans sa jeunesse il avait aimé feuilleter les pièces du chartrier, notamment celles témoignant des droits de son saint patron versés dans l'oubli ou le mépris. Fréquenter le chartrier, c'était déjà faire œuvre de piété et de dévotion envers saint Denis. Cette familiarité ancienne d'avec les archives, affichée complaisamment par Suger, n'avait rien d'exceptionnel: des abbés contemporains de l'abbé de Saint-Denis - tels Gautier de Saint-Amand ${ }^{7}$ ou Hariulf de Saint-Riquier ${ }^{8}$ dans le royaume de France, ou bien Léon du Mont-Cassin, Giovanni de Saint-Vincent au Volturne ou Oldrius de Casauria en Italie centrale ${ }^{9}$ - ont comme lui soulevé la poussière des chartes avant d'être promus à l'abbatiat, et ont fait même beaucoup plus que lui, en élaborant cartulaires ou chroniques-cartulaires, ce que Suger $n$ 'a pas entrepris, du moins dans ses années de jeunesse ${ }^{10}$.

»immunitates« ou Saint-Denis dans l'Église et dans l'État, dans: Bibliothèque de l'École des chartes 87 (1926), p. 20-97 et surtout p. 245-346.

6 L'ouvre, qui consiste en de véritables mauto-Gesta «, est traditionnellement désignée sous le nom de Liber de rebus in administratione sua gestis; le titre de Gesta Suggerii abbatis a été adopté par F. Gasparri. L'expression mauto-Gesta《 est d'Olivier GUYOTJEANNIN, La tradition de l'ombre: les actes sous le regard des archivistes médiévaux (Saint-Denis, $\mathrm{XII}^{e}-\mathrm{XV}^{\mathbf{e}}$ siècle), dans: Adam J. KoSTo, Anders WINROTH (dir.), Charters, Cartularies, and Archives: The Preservation and Transmission of Documents in the Medieval West. Proceedings of a Colloquium of the Commission Internationale de Diplomatique (Princeton and New York, 16-18 septembre 1999), Toronto 2002 (Papers in Mediaeval Studies, 17), p. 81-111, à la p. 97.

7 Gautier, abbé de Saint-Amand au diocèse de Tournai (1121-1124), est probablement l'auteur du premier cartulaire de l'abbaye en 1117; voir Henri PLATELLE, Le premier cartulaire de l'abbaye de Saint-Amand, dans: Le Moyen Âge 62 (1956), p. 301-329, à la p. 312.

${ }^{8}$ Hariulf $(\dagger 1143)$, moine de Saint-Riquier et auteur de la chronique-cartulaire du monastère (1088 et 1104) fut promu en dehors de son établissement d'origine, à Oudenbourg (Flandre) en 1105; voir Ferdinand LoT, introduction à Hariulf, Chronique de l'abbaye de SaintRiquier $\left(V^{e}\right.$ siècle-1104), Paris 1894 (Collection de textes pour servir à l'étude et à l'enseignement de l'histoire), p. V-IX.

9 Léon d'Ostie, Giovanni et Oldrius ont fait leurs preuves comme chroniqueurs-cartularistes avant de devenir abbés de leur monastère; cf. Laurent FELLER, Les Abruzzes médiévales: territoire, économie et société en Italie centrale du IX' au XII' siècle, Rome 1998 (Bibliothèque des écoles françaises d'Athènes et de Rome, 300), p. 72.

${ }^{10}$ Il est possible en effet que Suger ait fait faire un cartulaire dans les dernières années de sa vie, un recueil dont la matière serait passée dans les premières parties du "Livre des privilèges« du XIII siècle (Paris, Arch. nat., LL 1156): Rolf GROSSE, Remarques sur les cartulaires de Saint-Denis aux XIII et XIV siècles, dans: Olivier GUYOTJEANNIN, Laurent MORELLE, Michel PARISSE (dir.), Les cartulaires. Actes de la table-ronde organisée par I'École nationale des chartes et le G.D.R. 121 du C.N.R.S. (Paris, 5-7 décembre 1991), Paris 1993 (Mémoires et documents de l'École des chartes, 39), p. 279-288, aux p. 283-284. 
Une bonne connaissance du chartrier était un atout de poids pour un moine ambitionnant la charge abbatiale ${ }^{11}$. Les archives lui donnaient de la profondeur de champ, une largeur de vues et un ancrage "concret«, toutes choses utiles pour mettre en forme la politique qu'un futur prétendant à l'abbatiat pouvait nourrir pour son établissement. Bref, si Suger eut jamais une "certaine idée de Saint-Denis«, nul doute que les archives y contribuèrent. Il convient alors d'être sûr de bien entendre ce que nous dit l'abbé quand il parle d'elles.

Je m'intéresserai ici à deux passages célèbres du De administratione (début du chapitre 3 et fin du chapitre 20); l'un et l'autre, mais surtout le premier, ont été abondamment cités, traduits et commentés. Pourtant, ils me paraissent mériter un nouvel effort d'élucidation en raison des difficultés, de traduction ou d'interprétation, qu'ils présentent. Si le souci philologique ou lexicographique ne convainc pas le lecteur du bien fondé de l'enquête dans le cadre de cette rencontre, $\mathrm{j}$ 'ajouterai pour ma défense que mon propos touche quelques thèmes plus directement pertinents, comme les influences littéraires qui ont pesé sur les écrits de Suger, ou bien la conception que celui-ci avait de la mémoire archivistique dionysienne, voire les relations de Saint-Denis avec le pouvoir royal. J'espère alors être lavé du soupçon d'incongruité.

\section{Le chapitre 3 (De recuperatione Argentoilensis abbatiae)}

Le premier passage, fameux, ouvre le récit par lequel Suger raconte comment, en 1129 , il réussit à recouvrer le monastère d'Argenteuil. C'est, on l'a dit, l'occasion pour Suger de relater que dès sa jeunesse, il consultait souvent l'armarium de l'abbaye pour y glaner de quoi lutter contre de nombreuses revendications malhonnêtes. Le texte se présente ainsi ${ }^{12}$ :

Cum etate docibili adolescentiae meae antiquas armarii possessionum revolverem cartas, et immunitatum biblos propter multorum calumniatorum improbitates frequentarem, crebro manibus occurrebat de cenobio Argentoilensi fundationis carta ab Hermenrico et conjuge ejus Numma, in qua continebatur quod a tempore Pipini regis beati Dyonisii abbatia extiterat. Sed quadam occasione contractus incommodi, in tempore Karoli Magni filii ejus, alienata fuerat.

"FELLER (voir n. 9), p. 72, observe cependant que ce ne sera plus le cas à la fin du XII" siècle.

12 Suger, Gesta Suggerii abbatis, éd. GASPARRI (voir n. 1), p. 64. 
Madame Gasparri en a proposé la traduction suivante ${ }^{13}$ :

"Tandis que dans l'âge studieux de mon adolescence je compulsais les anciennes chartes de possession [conservées dans le] dépôt d'archives et que j'étudiais les registres d'immunités, en raison des manœuvres malhonnêtes de nombreux fraudeurs, il me tombait souvent entre les mains la charte de fondation du monastère d'Argenteuil par Hermenric et son épouse Numma: il y était écrit que dès le temps du roi Pépin l'abbaye avait appartenu à SaintDenis. Mais à l'occasion d'une convention désavantageuse, au temps de Charlemagne son fils, elle avait été aliénée.«

Ce passage suscite maintes interrogations. On aimerait naturellement éprouver la véracité du témoignage autobiographique et savoir si Suger consultait les chartes de son monastère dans le cadre d'un travail d'ordre archivistique. Répondre à cette question est cependant impossible pour le moment. Seule une enquête systématique à travers les mentions dorsales des originaux dionysiens, confrontant les chartes antérieures à l'abbatiat de Suger à celles contemporaines de son gouvernement, permettrait peut-être de saisir les éventuelles traces que le jeune moine a laissées sur les documents qu'il lisait ou classait dans ses années d'apprentissage ${ }^{14}$. Si le XII ${ }^{\mathrm{e}}$ siècle a connu une ou plusieurs campagnes d'inscription, notamment sur de nombreux actes mérovingiens et carolingiens, il est bien délicat, et encore trop tôt de toute façon, pour pouvoir en fixer le moment avec une précision satisfaisante, a fortiori pour les imputer au futur abbé de Saint-Denis ${ }^{15}$. On laissera donc cette question pour en aborder d'autres, susceptibles de recevoir des éléments de réponse.

${ }^{13}$ Ibid. p. 65.

${ }^{14}$ Sur les mentions dorsales dionysiennes en général: GUYOTJEANNIN (voir n. 6). - Pour les mentions de la fin du Moyen Âge: Donatella NEBBIAI-DALLA GUARDA, La bibliothèque de l'abbaye de Saint-Denis en France du IX $^{\mathfrak{e}}$ au XVIII ${ }^{\mathfrak{e}}$ siècle, Paris 1985 (Documents, études et répertoires publiés par l'Institut de recherche et d'histoire des textes), p. 78-79 et 118120.

${ }^{15}$ La recension exhaustive des mentions dorsales n'existe que pour les originaux antérieurs à 800 , grâce à l'édition (sans fac-similé des mentions toutefois) procurée par Hartmut ATSMA et Jean VEZIN, Chartae latinae antiquiores. Facsimile-edition of the latin charters prior to the ninth century, t. 13-17 (= France 1-5), Zurich 1981-1986; voir aussi Hartmut ATSMA, Le fonds des chartes mérovingiennes de Saint-Denis, rapport sur une recherche en cours, dans: Paris et Île-de-France. Mémoires publiés par la Fédération des sociétés historiques et archéologiques de Paris et d'île-de-France 32 (1981), p. 259-272, à la p. 261 et p. 269, n. 25 (12 originaux ont des mentions pouvant remonter au XII $\mathrm{s}$.). Grâce à l'amical truchement de Rolf Große, j'ai obtenu de Jens Peter Clausen - qui voudra bien trouver ici la marque de ma gratitude -, de précieux renseignements sur la morphologie des notes dorsales inscrites au XII ${ }^{\mathrm{e}}$ siècle sur les actes mérovingiens et carolingiens. 


\section{Suger a-t-il consulté la charte de fondation du monastère d'Argenteuil?}

Le texte constitue une pièce de choix dans le dossier épineux des premiers temps de l'abbaye féminine d'Argenteuil et de son éventuelle soumission à Saint-Denis dès l'époque mérovingienne. Par là, il touche à la question controversée des forgeries dont Suger se serait éventuellement rendu coupable pour faire prévaloir les prétentions dionysiennes devant le concile réuni à Saint-Germain des Prés en février-mars $1129^{16}$. Je ne compte rouvrir ici ce dossier que pour faire quelques observations strictement liées au passage cité.

Suger y déclare que dans sa jeunesse, il a vu dans le chartrier dionysien la charte par laquelle Ermenricus et son épouse Numma (sic pour Mumma) avaient fondé le monastère d'Argenteuil. C'est la seule attestation que l'on ait de l'existence matérielle de cette pièce d'archives. Selon toute vraisemblance, Suger ne l'a pas produite lors de la réunion de 1129; la preuve décisive exhibée à cette occasion par Suger fut en effet un précepte de Louis le Pieux et Lothaire de $[828]^{17}$, comme l'atteste clairement le diplôme confirmatif de Louis VI issu de cette instance, un acte royal d'ailleurs rédigé par un moine dionysien sur le modèle du diplôme carolingien ${ }^{18}$. Il est vrai toutefois que le

${ }^{16}$ Thomas G. Waldman, Abbot Suger and the nuns of Argenteuil, dans: Traditio 41 (1985), p. 239-272 (trad. partielle par Jean DUFOUR, L'abbé Suger et les nonnes d'Argenteuil, dans: Le Vieil Argenteuil 29 [1986-1987], p. 5-26); Josef SEMMLER, Saint-Denis: Von der bischöflichen Coemeterialbasilika zur königlichen Benediktinerabtei, dans: Hartmut ATSMA (dir.), La Neustrie: Les pays au nord de la Loire de 650 à 850 , colloque historique international, Sigmaringen 1989 (Beihefte der Francia, 16), t. 2, p. 75-123, aux p. 113-115; JeanLouis BERNARD, Argenteuil (Val d'Oise): état des connaissances sur l'abbaye Notre-Dame de sa fondation à l'époque romane, dans: Les Cahiers de Saint-Michel de Cuxa 23 (1992), p. 149-151; Jean DufouR (éd.), Recueil des actes de Louis VI, roi de France (1108-1137), 4 vol., Paris 1992-1994 (Chartes et diplômes relatifs à l'histoire de France), t. 2, p. 102-103 (note critique du diplôme royal $n^{\circ} 281$ du 14 avril 1129 confirmant les droits de Saint-Denis sur Argenteuil); Ralf PETERS, Die Entwicklung des Grundbesitzes der Abtei Saint-Denis in merowingischer und karolingischer Zeit, Aix-la-Chapelle 1993, p. 87-88; Rolf GrossE (éd.), Papsturkunden in Frankreich, Neue Folge, t. 9. Diözese Paris, 2: Abtei Saint-Denis, Göttingen 1998 (Abhandlungen der Akademie der Wissenschaften in Göttingen, philologisch-historische Klasse, 3. Folge, 225), p. 141-144 (note critique développée relative au document $n^{\circ} 32$ [févr.-mars 1129], acte du légat Matthieu d'Albano promulguant le jugement de l'assemblée réunie à Saint-Germain des Prés).

${ }^{17}$ Johann Friedrich BÖHMER, Engelbert MOHLBACHER, Regesta Imperii, t. 1: Die Regesten des Kaiserreichs unter den Karolingern 751-918, Hildesheim 1966 ( ${ }^{\circ}$ éd., Innsbruck 1908), $n^{0} 848$, p. 332, acte conservé en copie; éd. WaLdMAN (voir n. 16), p. 266-271 et Jean DUfOUR, Les plus anciens actes pour Argenteuil, IV. Précepte de Louis le Pieux et de Lothaire, dans: Le Vieil Argenteuil 35 (1998-99), p. 5-9.

${ }^{18}$ Éd. DUFOUR, Recueil (voir n. 16), t. 2, $\mathrm{n}^{\circ}$ 281, p. 100-106 (sur le diplôme impérial comme modèle du diplôme de Louis VI, voir la note critique, ibid. p. 102-103). Peut-on savoir quelles pièces diplomatiques Suger a présentées devant l'assemblée de Saint-Germain des Prés? Dans son acte (cf. le passage du diplôme royal, p. 105, l. 1-7), le roi (présent à l'assemblée) précise que Suger lui a présenté un diplôme (preceptum) impérial, celui de Louis le Pieux et Lothaire, dont la teneur est décrite en détail. Mais dans la phrase qui suit, 
diplôme de [828] mentionne la charte d'Hermenricus et de Mumma, en précisant que par cet instrument, le couple avait fondé le monastère féminin d'Argenteuil sur ses propres terres et en avait fait don à Saint-Denis; outre cette charte, le diplôme impérial mentionne la confirmation de la donation par un roi Clotaire qui ne peut être que Clotaire III $[657-673]^{19}$. Selon Thomas Waldman, ces deux actes mérovingiens seraient au mieux des faux, au pire de simples »fantômes" diplomatiques inventés par Suger pour en faire état dans le diplôme de [828], lui-même par conséquent falsifié voire forgé par les soins de l'abbé ${ }^{20}$. Cette opinion toutefois n'a pas fait l'unanimité des spécialistes ${ }^{21}$. À la vérité, et sans vouloir engager un examen approfondi qui n'a pas sa place ici, l'existence et la teneur des deux actes mérovingiens n'ont rien d'extra-

le rédacteur emploie à son sujet un pluriel ( $Q$ uibus coram sepius inspectis religiosarum personarum), peut-être simplement induit par la description du diplôme impérial qui venait juste avant, où l'on évoquait un précepte de Clotaire III (sur ce deperditum, voir ci-dessous, n. 19). Quant à la sentence du légat Matthieu d'Albano (éd. Grosse [voir n. 16], $\mathrm{n}^{\circ} 32$, p. 141-144, à la p. 144), elle emploie aussi un pluriel au sujet des preuves alléguées par Suger, mais dans un contexte vague: Suggerius, emunitatibus suis apostolicorum confirmatione certissimis in medium ostensis, prefatum monasterium ad ius ecclesie sue pertinere satis evidenter ostendit. R. Große identifie ces emunitates d'une part avec le diplôme de Louis le Pieux et Lothaire, d'autre part avec le précepte déjà cité de Clotaire III mentionné par le diplôme impérial.

${ }^{19}$ Qui [= Hilduinus abbas] statim donationem cujusdam Deum timentis ac religiosi hominis, nomine Ermendrici, ac conjugis sue Mummane, qui prefatum monasterium in suo proprio construxerant, et id per testamenti paginam ad beati Dyonisii, martyris Christi, monasterium sollempni donatione contulerant, necnon et preceptum confirmationis Hlotharii, quondam regis, quod super eandem donationem conscribere jusserat, nobis ad relegendum ostendit (éd. WALDMAN [voir n. 16], p. 268; éd. DUFouR [voir n. 17], p. 8). - Sur le deperditum de Clotaire III: Die Urkunden der Merowinger, éd. Carlrichard BROHHL, Theo KOLZER, coll. Martina HARTMANN, Andrea STIELDORF, 2 vol., Hanovre 2001 (MGH, Diplomata regum e stirpe Merowingica), t. 2, p. 605-606, dep. $n^{\circ} 252$ (notice imputable à A. Stieldorf), jugé "nzweifelhaftu.

${ }^{20}$ Selon WALDMAN (voir n. 16), p. 256, Suger aurait emprunté les deux noms d'Ermenricus et de Mumma à la charte de fondation (673) du monastère de Bruyères par Clotilde, conservée en original dans le fonds de Saint-Denis; sur cet acte, voir Léon LEviLLAIN, Études mérovingiennes. La charte de Clothilde (10 mars 673), dans: Bibliothèque de l'École des chartes 105 (1944), p. 5-63. Suger aurait ensuite injecté la mention des deux fondateurs et de leur charte, ainsi que celle de la confirmation de Clotaire III, dans le diplôme de Louis le Pieux et Lothaire.

${ }^{21}$ Les arguments de Waldman ont convaincu DufouR, Recueil (voir n. 16), t. 2, p. 102-103, mais nullement SEMMLER (voir n. 16), p. 113, n. 4, ni GROSSE (voir n. 16), p. 142-143, pour lesquels les arguments apportés contre la véracité du diplôme de [828] sont bien trop vagues. A. STIELDORF (Urkunden der Merowinger [voir n. 19], t. 2, p. 606) se contente de dire que J. Semmler n'a pas apporté de contre-arguments à ceux de Th. Waldman. L'authenticité du diplôme de [828] vient d'être également défendue par Françoise GASPARRI, La politique de l'abbé Suger de Saint-Denis à travers ses chartes, dans : Cahiers de civilisation médiévale 46 (2003), p. 233-245, aux p. 242-243. 
vagant ${ }^{22}$ et même la sujétion qu'ils affirment du monastère d'Argenteuil à l'égard de Saint-Denis, pourtant si difficile à faire concorder avec certaines données de deux préceptes de 697 et $769^{23}$, n'est peut-être pas un obstacle infranchissable. On pourrait envisager, d'une part, que la soumission prévue par les fondateurs ne devait prendre effet qu'à la mort de l'abbesse en fonction en 697 - un cas de figure attesté pour d'autres monastères »mineurs « gravitant dans l'orbite d'abbayes de première grandeur ${ }^{24}$-, d'autre part, que l'autonomie d'Argenteuil fut reconquise au siècle suivant, avant 769. En tout état de cause, on voit qu'une nouvelle expertise du diplôme de [828] et de ses données serait bienvenue.

Ce que le De administratione nous dit de la charte d'Ermenricus-Mumma invite toutefois à douter sérieusement que Suger l'ait jamais eu entre les mains. On peut déjà s'étonner que Suger prétende avoir vu la charte des fondateurs d'Argenteuil alors que c'est l'acte confirmatif de Louis le Pieux et Lothaire, dont il ne dit mot ici, qui fut la pierre d'angle de son argumentaire de 1129. Il est difficile, cependant, d'aller au-delà du simple constat, car on ne peut prendre Suger en défaut dans le texte même du De administratione. L'abbé poursuit en effet son histoire en déclarant avoir envoyé à Rome, à l'appui de ses revendications, "nos messagers avec les anciennes chartes de fondation et de donation, et les privilèges de confirmation ${ }^{25}$; cette déclaration est assurément floue et générale, comparée à la précision analytique de notre passage, mais elle ne la contredit pas.

${ }^{22}$ La fondation d'Argenteuil par le couple Ermenricus-Mumma est loin d'être historiquement aberrante: le rapprochement onomastique avec les données de la charte de Clotilde de 673 (voir ci-dessus, $n$. 20) pourrait s'expliquer par le fait que ces personnages gravitaient dans l'orbite de Saint-Denis.

${ }^{23}$ Je fais ici allusion aux deux diplômes délivrés par les rois Childebert III (697) et par Carloman (769) en faveur des moniales d'Argenteuil. Ces deux préceptes originaux (éd. ATSMA, VEZIN [voir n. 15], t. 17, Zurich 1984, $n^{\circ} 654$, p. 60-63 [Childebert III] et $n^{\circ} 658$, p. 82-85 [Carloman]), portant l'un concession du bois de Comioletus, l'autre concession d'immunité, sont adressés à l'abbesse d'Argenteuil sans jamais citer l'abbaye de Saint-Denis ni faire allusion, de près ou de loin, à quelque sujétion d'Argenteuil à l'égard de quelqu'autre établissement.

${ }^{24}$ Voir l'exemple du monastère féminin d'Honnecourt (en Cambrésis), dont le fondateur Amalfridus prévoit en 687 l'entrée de sa fondation dans la dépendance de l'abbaye de SaintBertin, mais cette sujétion n'est destinée à devenir effective qu'à la mort de l'abbesse Auria(na), sa fille; voir Maurits GYSSELING, Anton C. F. KOCH (éd.), Diplomata Belgica ante annum millesimum centesimum scripta, t. 1, Bruxelles 1950, ${ }^{\circ} 5$, p. 15-17 (donation d'Honnecourt à Saint-Bertin par Amalfridus, 687) et $n^{\circ}$ 6, p. 17-18 (confirmation de la donation par Thierry III, 689, acte jugé faux par KÖLZER, Urkunden der Merowinger [voir n. 19], t. $1, n^{\circ} 130$, p. $329-331$ ).

${ }^{25}$ Suger, Gesta Suggerii abbatis, éd. GASPARRI (voir n. 1), p. 64-65 (trad. de l'éditeur): nuncios nostros et cartas antiquas fundationis et donationis et confirmationum privilegia bonae memoriae papae Honorio Romam delegavimus ... On trouvera dans DUFOUR, Recueil (voir n. 16), t. 2, p. 102-104, un commode état des textes où Suger rapporte l'affaire d'Argenteuil. 
De la contradiction, il y en a pourtant, au cœur même du passage qui nous occupe. À lire Suger, la teneur de l'acte d'Ermenricus-Mumma est proprement aberrante: la charte ne pouvait en aucune manière raconter qu'Argenteuil était une abbaye soumise à Saint-Denis »depuis le temps du roi Pépin [751-768]«. Comment en effet Suger peut-il admettre qu'Argenteuil aurait été fondée et donnée à Saint-Denis à l'époque du père de Charlemagne, alors que le diplôme de [828], fort bien connu de Suger, invoquait une confirmation de la donation par un roi Clotaire, nécessairement mérovingien? L'hypothèse, avancée par Josef Semmler, d'une confusion entre Pépin II de Herstal et Pépin III le Bref me paraît invraisemblable car on ne voit pas comment et à quel titre la charte d'Ermenricus-Mumma, qui date au plus tard des années [657-673], aurait pu contenir le nom de Pépin II, dont la latitude d'action en Neustrie ne peut être antérieure à la victoire de Tertry $(687)^{26}$.

Le passage, dont la latinité est curieusement gauche par endroit ${ }^{27}$, présente une autre bizarrerie. Rapportant la teneur de l'acte d'Ermenricus-Mumma, Suger déclare que l'abbaye d'Argenteuil était soumise à Saint-Denis »à partir de Pépin« (a tempore Pipini regis). Cette tournure laisse entendre que le statut inauguré sous Pépin s'est prolongé au moins quelque temps après ce roi. Or, dans la phrase suivante, l'abbé affirme qu'Argenteuil a été aliénée »au temps de Charlemagne, son fils«, donc aussitôt après. L'expression a tempore Pipini ne semble donc guère appropriée. Serait-elle le fruit d'une corruption textuelle?

${ }^{26}$ SEMMLER (voir n. 16), p. 114. Du passage en cause de Suger, l'historien tire l'hypothèse aventureuse selon laquelle c'est sous Pépin II qu'Argenteuil aurait regagné son autonomie. Outre l'identification très problématique de Pépin, cette hypothèse revient à faire dire à Suger l'inverse de ce qu'il dit, puisque c'est à partir de Pépin que, selon Suger, Argenteuil fut soumis à Saint-Denis. Selon GASPARRI (voir n. 1), p. 196, n. 29, Suger aurait commis une double confusion: Pépin III au lieu de Pépin II et Charlemagne au lieu de Carloman. Ces transformations me semblent excéder la prudence.

${ }^{27} \mathrm{La}$ façon d'exprimer la sujétion d'Argenteuil (in qua continebatur quod a tempore Pipini regis beati Dyonisii abbatia extiterat) me paraît curieuse. Que l'on comprenne littéralement: "... il (le coenobium d'Argenteuil) avait été (extiterat) abbaye (abbatia) de Saint-Denis (= relevant de Saint-Denis)«, ou bien: „l'abbaye (abbatia, c'est-à-dire l'abbaye d'Argenteuil) était de Saint-Denis «, on reste un peu gêné devant cet emploi d'exsistere, là où on attendrait plutôt un juris esse. N'était son absurdité (Saint-Denis passait pour une fondation de Dagobert $\mathrm{I}^{\mathrm{er}}$ ), la traduction obvie du passage serait plutôt: "... que l'abbaye de Saint-Denis avait existé depuis l'époque du roi Pépin.« $R$. Große, qui m'a fait l'amitié de regarder le texte avec moi, suggère que le texte primitif présentait un génitif abbatie corrigé en abbatia pour éviter la succession de cinq génitifs. - Sur le latin de Suger: Françoise GASPARR, Le latin de Suger, abbé de Saint-Denis (1081-1151), dans: Monique GouLLET, Michel PARISSE (dir.), Les historiens et le latin médiéval. Colloque tenu à la Sorbonne les 9 , 10 et 11 septembre 1999, Paris 2001 (Histoire ancienne et médiévale, 63), p. 177-193; cf. aussi Jean-Yves TILLIETTE, La place de la Vie de Louis le Gros par Suger dans l'histoire de la biographie royale, dans: Dorothea WALZ (dir.), Scripturus Vitam. Lateinische Biographie von der Antike bis in die Gegenwart. Festgabe für Walter Berschin zum 65. Geburtstag, Heidelberg 2002, p. 979-987. 
Toutes ces scories ${ }^{28}$, maladresses voire énormités qu'on repère dans le passage du De administratione plongent le lecteur dans l'embarras. Peut-être balaiera-t-on ces difficultés d'un revers de main en objectant qu'à l'époque où il rédigeait son ouvrage, à partir de $1144^{29}$, Suger n'avait plus en tête le dossier d'une affaire déjà vieille de trois lustres. Certes, on peut éprouver l'incidence de l'éloignement du temps dans sa Vita Ludovici, une œuvre qui date des années $1137-1143^{30}$. Quand on y lit le passage consacré par l'abbé aux actes royaux qu'il avait présentés à Louis VI pour étayer ses revendications, on s'aperçoit que les diplômes cités ne renforçaient pas tous, bien au contraire, l'argumentaire dionysien, puisque l'un d'entre eux, le précepte de Carloman, témoignait en faveur de l'autonomie d'Argenteuil ${ }^{31}$. En réalité, ces pièces avaient pour seuls points communs de mentionner Argenteuil et d'émaner de souverains: devant un tel récit, on se prend à imaginer que Suger égrenait les pièces d'autorité renfermées dans le scrinium relatif à ce monastère, sans trop se soucier de leur adéquation à la cause jugée en 1129. La sentence était ancienne, notre chroniqueur s'estimait peut-être affranchi des contraintes qui s'étaient imposées à l'avocat! Je ne suis pas sûr, néanmoins, qu'il en aille de même pour notre passage du De administratione. Suger n'y énumère pas »en vrac « des noms de souverains auteurs de diplômes, il produit comme l'»analyse diplomatique« d'une pièce bien ciblée. Par conséquent, la distance chronologique ne suffit pas, à mon sens, à expliquer les aberrations et anomalies qu'on y a détectées.

L'irruption du nom de Pépin dans le passage en cause nous met peut-être sur une piste, celle du diplôme de [828], encore lui. De la lecture de cet acte, en effet, on déduit que la sujétion d'Argenteuil s'était maintenue jusqu'à l'époque précédant immédiatement Charlemagne, donc jusqu'au règne de son père Pépin. Et le même diplôme offre aussi matière à parler d'un contractus incommodus passé sous Charlemagne, puisqu'il rappelle que l'empereur avait cédé Argenteuil à sa fille Théodrade. Tout se passe par conséquent comme si Suger, bien que parlant de la charte d'Ermenricus-Mumma, n'avait en tête (et en vue) que le précepte de Louis le Pieux et Lothaire de [828] mentionnant cette charte. On pourrait alors imaginer que l'abbé avait d'abord choisi de décrire le

${ }^{28}$ Rappelons aussi la faute Numma pour Mumma.

${ }^{29}$ GASPARRI (voir n. 1), p. LV-LVI.

${ }^{30}$ Ibid. p. LIV.

${ }^{31}$ Vita Ludovici Grossi regis, éd. et trad. Henri WAQUET, Suger, Vie de Louis VI le Gros, $2^{\mathrm{e}}$ éd., Paris 1964 (Les classiques de l'histoire de France au Moyen Âge, 11), p. 216: Qui [= Louis VI], cum justiciam nostram [= celle de Saint-Denis] de monasterio Argentoilensi ... cognovisset, precepta regum antiquorum Pipini, Karoli Magni, Ludovici Pii et aliorum de jure loci prefati nunciis nostris oblata perlegisset, curie tocius persuasione ... beato Dyonisio ... et restituit et confirmavit. Pour l'identification des actes évoqués (Pépin de 768, Carloman de 769, Louis le Pieux et Lothaire de [828]), voir Dufour, Recueil (voir n. 16), t. 2, p. 103-104, n. 17 et 12. Sur le diplôme de Carloman, voir ci-dessus n. 23. 
diplôme, puis qu'il s'est ravisé, préférant parler de la charte, en oubliant cependant d'apporter au texte toutes les rectifications nécessaires. Dans cette hypothèse, il est toujours loisible de proposer quelques retouches, aux fins de reconstituer l'agencement présumé primitif ${ }^{32}$. Mais cet acharnement philologique est un peu vain. Mieux vaut sans doute retenir que les incohérences de fond et de forme signalent probablement, de la part de Suger, une confusion née du fait qu'il travaillait en réalité sur le seul diplôme de [828].

On conclura donc de ce premier examen que Suger n'est précis qu'en apparence, qu'il n'a probablement pas vu la charte d'Ermenricus-Mumma dont il parle et que les éléments d'analyse qu'il en donne dérivent au fond du diplôme impérial de [828], qui constituait assurément le fondement de ses revendications sur Argenteuil.

\section{Que signifie le terme »biblos»?}

Ce passage du De administratione va nous retenir à présent pour une question d'ordre lexicographique. Il s'agit d'explorer l'expression biblos immunitatum. Si Marcel Aubert l'a prudemment laissée telle quelle ${ }^{33}$, Françoise Gasparri l'a rendue par wregistres d'immunités«, Thomas Waldman a adopté l'expression "collections of immunities ${ }^{34}$ et Jean Dufour celle de "cartulaires «; enfin, Jean Schneider et Michel Bur ont adopté l'expression »recueils de privilèges « ${ }^{35}$. Si on néglige les nuances entre les termes, toutes les traductions adoptées ren-

32 On pourrait ainsi changer l'expression quod a tempore Pippini en une formule du genre quod usque ad tempus Pippini, et le passage occurrebat de cenobio Argentoilensi fundationis carta ab Hermenrico et conjuge ejus Numma, in qua pourrait être ainsi modifié: occurrebat de cenobii Argentoilensis fundatione ab Hermenrico et conjuge ejus Numma carta, in qua ...

${ }^{33}$ Marcel AUBERT, Suger, Saint-Wandrille 1950 (Figures monastiques), p. 4, n. 14, cite la phrase en question, sans la traduire ni s'attarder sur biblos immunitatum. Le commentaire de la note fait écho au revolverem cartas tandis que dans le texte, l'historien reste assez évasif: »Suger ... passait une partie de son temps au chartrier de Saint-Denis, à classer et à analyser les pièces d'archives ....

${ }^{34}$ WALDMAN (voir n. 16), p. 241-242: "When in the very impressionable age of my adolescence, I used to leaf through the ancient charters of the abbey's possessions kept in its chests, and since, because of the dishonest deeds of many persons guilty of fraud, I had to consult our collections of immunities ...«; trad. de DUFOUR, L'abbé Suger (voir n. 16), p. 7: "Comme... je lisais les vieux documents du chartrier [de Saint-Denis] et compulsais les cartulaires en raison des malhonnêtetés de nombreux revendicateurs ...\&

${ }^{35}$ Suger, La Geste de Louis VI et autres cuvres. Présentation: Michel BuR, Paris 1994 (Acteurs de l'Histoire), p. 223: "Quand jeune et studieux, je feuilletais les chartes anciennes des archives et consultais les recueils de privilèges à cause des entreprises malhonnêtes de nombreux fraudeurs ... «; d'après les remerciements de la p. 43, la traduction est empruntée à un travail inédit de Jean Schneider. Même traduction dans Michel BUR, Suger, abbé de SaintDenis et régent de France, Paris 1991, p. 38. 
voient donc à la notion de livres rassemblant des actes. En somme, les biblos immunitatum seraient des recueils de copies mêlant des préceptes d'immunités et d'autres chartes conférant divers droits et privilèges de l'abbaye ${ }^{36}$.

Suger décrirait donc les archives de son monastère en constatant qu'elles ne comportaient pas seulement des originaux mais aussi des recueils de copies d'actes. Une telle description des archives serait beaucoup moins banale qu'il n'y paraît. Les textes de nature historiographique parlent vaguement des archives, sans ce souci "technique« que les traductions prêtent à l'abbé. On pourrait même avancer par une glose hardie que pour Suger, les cartulaires mêlés indistinctement aux chartes avaient une valeur (d'usage en tout cas) équivalente à celle des originaux. Bref, nous serions avec Suger dans le neuf et l'original.

L'interprétation est séduisante mais je la crois très fragile pour la raison que les traductions proposées pour biblos immunitatum me semblent en réalité fort douteuses. En traduisant le terme biblos par des mots englobant l'idée de »livre«, on le rapproche de son parent tardif biblion, qui appartient au latin chré$\operatorname{tien}^{37}$, alors que biblos, plus anciennement attesté, signifie "papyrus $\aleph^{38}$. La notice »biblus« du »Mittellateinisches Wörterbuch« signale d'ailleurs que le sens de »livre« est essentiellement poétique et qu'il disparaît à partir du $\mathrm{XI}^{\mathrm{e}}$ siècle $^{39}$. Niermeyer n'a pas fait apparaitre le mot dans son "Lexicon minus«, ce qui veut dire que le vocable a conservé à ses yeux son sens classique de "papyrus « ou d'»écrit sur papyrus ${ }^{40}$. Le lexicographe a cependant introduit l'adjectif bibleus, auquel il donne le sens d'»écrit sur papyrus« et pour lequel il cite un texte hagiographique de la fin du $\mathrm{XI}^{\mathrm{e}}$ siècle tout-à-fait clair ${ }^{41}$. De fait, le sens de "papyrus« pour biblos est clairement attesté dans une œuvre légèrement postérieure à celle de Suger, la chronique du monastère alémannique de Petershausen. L'auteur, qui rédige après $1156^{42}$, y désigne, par ce terme au

${ }^{36}$ On ne sait trop en effet si immunitas est à prendre dans une acception carolingienne ou si, comme c'est plus probable, il recouvre toutes sortes d'exemptions, locales ou générales, laïques ou ecclésiastiques.

${ }^{37}$ Pierre FLOBERT (dir.), Le Grand Gaffiot: Félix Gaffiot. Dictionnaire latin-français, Paris 2000 , p. 219 , s. v. biblion, $-i i, \mathrm{n}$.

${ }^{38}$ Biblos ou biblus, -i; le mot est féminin. Cf. Le Grand Gaffiot (voir n. 37), p. 219.

${ }^{39}$ T. 1: A-B, par Otto PRINZ, Johannes SCHNEIDER, Munich 1967, col. 1464.

${ }^{40}$ GASPARRI (voir n. 1), p. 255 (glossaire), donne pour biblus les sens suivants: »écrit, papier, livre«. Le choix de traduction fait dans l'édition n'est donc pas le plus heureux.

${ }^{41}$ Jan Frederik NIERMEYER, Mediae latinitatis lexicon minus, achevé par C. VAN DE KIEFT, Leyde 1976, p. 97. Il s'agit d'un passage des Miracula s. Liutwini [ep. Treverensis] auctore monacho Mediolacensi (= Mettlach), éd. Heinrich Volbert SAUERLAND, dans: $\mathbf{M G H}$, Scriptores, t. 15/2, Hanovre 1888, p. 1261-1268, chap. 11, p. 1265: Quin eciam a Roma, quo ipse [ $=$ Robert, archevêque de Trèves, $\uparrow 956]$ ad apostolorum principem pro suorum salute venerat, apostolice benedictionis munus bible is cartis inscriptum Mediolacensibus detulerat, in quibus apostolicus requiem defunctis preobtavit ...

42 Casus monasterii Petrishusensis, éd. Otto ABEL, Ludwig WEILAND, dans: MGH, Scriptores, t. 20 , Hanovre 1868 , p. 624-682; sur l'œuvre, voir les références rassemblées par Hans 
pluriel (biblis), le support sur lequel était inscrit le texte du privilège de protection apostolique délivré par le pape Jean XII (Jaffé-Loewenfeld $n^{\circ} 3831$ ) au monastère le 25 avril $989^{43}$. Plus proche de Saint-Denis, un exemple fort semblable est donné par les sources issues de l'abbaye de Fleury. Dans une lettre qu'il adresse au pape Grégoire V (996-999), Abbon de Fleury ( $†$ 1004) supplie le pontife »de suivre la tradition de ses prédécesseurs en validant, en faveur de nouveaux monastères, de nouveaux écrits en forme de mandements (novos biblos indiculorum) «, afin d'épargner aux communautés »le vacarme du monde ${ }^{44}$. En d'autres termes, Abbon demande au pape de délivrer des privilèges assurant aux monastères la protection apostolique. Dans cette expression, le terme indiculus est assez recherché, mais s'est ménagé une place dans le vocabulaire diplomatique depuis l'époque mérovingienne; il insiste sur la nature de »message« de l'acte sollicité ${ }^{45}$. Quant au terme biblos, non seule-

Werner GOETZ, Zum Geschichtsbewußtsein in der alamannisch-schweizerischen Klosterchronistik des hohen Mittelalters (11.-13. Jahrhundert), dans: Deutsches Archiv 44 (1988), p. 459, n. 19.

${ }^{43}$ Privilegium monasterii in biblis primitus scriptum, quod et hactenus est in monasterio conservatum [rubrique]. Hoc est privilegium quod B. Gebehardus a Romano pontifice Joanne super locum ipsum percepit [suit le texte de l'acte] (Casus [voir n. 42], chap. 27, p. 633). Pour Harry BRESSLAU, Papyrus und Pergament in der päpstlichen Kanzlei bis zur Mitte des 11. Jahrhunderts, dans: Mitteilungen des Instituts für österreichische Geschichtsforschung 9 (1888), p. 1-33, à la p. 14, n. 4, la rubrique laissait entendre que l'original sur papyrus n'était plus conservé au moment de la rédaction de la chronique; ce qui veut bien dire que le savant diplomatiste traduisait naturellement in biblis par "sur papyrus«. Dans sa version des Casus, le privilège n'est pas entièrement sincère: cf. Harald ZIMMERMANN (éd.), Papsturkunden 896-1046, t. 1, Vienne 1984 (Österreichische Akademie der Wissenschaften, phil.-hist. Klasse, Denkschriften, 174), $\mathrm{n}^{\circ} 296$, p. 575 (note critique); voir aussi HansHenning KORTÜM, Zur päpstlichen Urkundensprache im frühen Mittelalter. Die päpstlichen Privilegien 896-1046, Sigmaringen 1995 (Beiträge zur Geschichte und Quellenkunde des Mittelalters, 17), p. 304, n. 364.

${ }^{44}$ Lettre III, dans: MIGNE PL, t. 139, col. 422C: Quapropter vestram serenitatem plus solito deprecor ut, praedecessorum morem sequendo, eisdem novis monasteriis novus auctor novos indiculorum biblos corroboretis, quibus ipsae congregationes ab omni mundano strepitu quietae Domino servire possint, maxime sub epitimio excommunicationis. On trouve exactement la même phrase dans une lettre d'Albert de Micy au pape Jean XVIII (PL 139, col. 440A): Dignum est enim, venerande pater, ut praedecessorum vestrorum morem sequamini, et monasteriis novos indiculorum biblos corroboretis, quibus ipsae congregationes ab omni ... etc.

${ }^{45}$ Cf. Olivier GuYOTJEANNIN, Le vocabulaire de la diplomatique en latin médiéval (noms de l'acte, mise par écrit, tradition, critique, conservation), dans: Olga WEIJERS (dir.), Vocabulaire du livre et de l'écriture au moyen âge, actes de la table ronde (Paris, 24-26 sept. 1987), Turnhout 1989 (Études sur le vocabulaire intellectuel du Moyen Âge, 2), p. 120-134, à la p. 122. La lettre d'Abbon ne permet pas de suivre jusqu'au bout l'auteur quand il affirme (p. 122, n. 8), sur la base de la Collectio canonum du canoniste, qu'Abbon réserve le terme d'indiculus aux actes de roi ou d'empereur. Sur le sens d'indiculus à l'époque mérovingienne, voir Peter CLASSEN, Kaiserreskript und Königsurkunde. Diplomatische Studien zum Problem der Kontinuität zwischen Altertum und Mittelalter, Thessalonique 1977 (Byzantina 
ment le contexte interdit de penser qu'il puisse signifier »livre«, mais il est patent qu'il a conservé, comme dans le cas de Petershausen, le sens primitif de "papyrus«: on sait qu'à l'époque d'Abbon, les actes pontificaux étaient ordinairement écrits sur un tel support ${ }^{46}$. L'ancien archiviste Abbon emploie donc le terme en fin connaisseur ${ }^{47}$.

Ces exemples invitent donc à conclure qu'à l'époque de Suger, les mots biblos et bibleus désignent le support sur papyrus ou la charte écrite sur papyrus. Peut-être fera-t-on observer que, dans tous les exemples développés, les termes s'appliquent à des productions de la chancellerie pontificale. Mais ce constat ne doit pas nous conduire à réduire le champ lexical des deux termes. Comme la chancellerie pontificale fut la dernière à abandonner l'usage du papyrus pour ses productions diplomatiques, beaucoup de monastères - dont Saint-Denis n'était pas - ne possédaient pas d'autres papyrus que des privilèges venus de Rome, ce qui ne veut pas dire que le terme biblos était réservé aux actes pontificaux.

Des développements précédents, il ressort que les bibli immunitatum signalés par Suger ne sont pas des »recueils d'immunités«, mais des "préceptes et privilèges écrits sur papyrus $\aleph^{48}$. Partant, la phrase en cause de Suger n'oppose plus les chartes originales aux livres de copies mais, plus traditionnellement, les chartes fondant les droits de Saint-Denis sur les possessions (cartae possessionum) aux actes d'autorité établissant les libertés de Saint-Denis (bibli

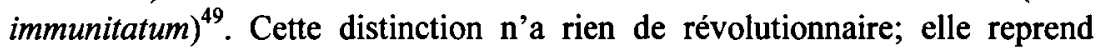
grosso modo l'opposition très ancienne, sensible dès l'époque franque, entre actes privés et actes d'autorité.

Nous ne perdons pas tout avec l'interprétation proposée. On peut penser, en effet, que le terme biblos n'est pas une pure coquetterie de style, destinée seulement à opérer une variation par rapport au mot carta, mais qu'il témoigne de

keimena kai meletai, 15), p. 144-145: indiculus s'applique à des lettres émanant aussi bien du roi que d'expéditeurs dépourvus d'autorité.

${ }^{46}$ Le premier acte pontifical sur parchemin qui soit conservé date de 967 (JAFFÉ-LOEWENFELD $n^{\circ} 3714$ ), le dernier sur papyrus qui soit attesté date de 1057 (JAFFÉ-LOEWENFELD $n^{\circ} 4366$ ); cf. BRESSLAU (voir n. 43), p. 11-13 et p. 29.

${ }^{47}$ Le terme biblos (ou biblus) n'est pas relevé par GUYOTJEANNIN (voir n. 45), mais sa pêche était déjà tellement abondante!

${ }^{48}$ Malgré son choix de traduction, F. Gasparri semble être gênée par le terme biblos, qu'elle cite volontiers tel quel; voir son étude: L'abbé Suger (voir n. 2), p. 255: "Nous savons, parce qu'il nous le dit lui-même, que dans son jeune âge, alors qu'il était encore écolier, il étudiait les anciennes chartes de l'abbaye, cartas, biblos, catalogos, ainsi que les édits impériaux, les anciens récits, les préceptes des rois qui sont, dit-il, déposés dans les archives publiques«".

49 Mon confrère et ami Jean Dufour m'a fait remarquer que biblos pourrait aussi évoquer plus spécialement des actes roulés. À cet égard, on rappellera qu'en 1844 encore, les actes dionysiens sur papyrus étaient conservés roulés, au témoignage d'Henri Bordier: cf. ATSMA (voir n. 15), p. 270 , n. 44. 
la conscience que les moines dionysiens avaient de la singulière abondance du chartrier de Saint-Denis en documents écrits sur papyrus. De cette richesse, il subsiste encore aujourd'hui treize chartes royales et quatre actes privés de l'époque mérovingienne ${ }^{50}$, auxquels les temps carolingiens ont ajouté pour le moins deux privilèges pontificaux et trois lettres sur le même matériau ${ }^{51}$. Mais dans les années 1060 , les faussaires dionysiens n'avaient pas craint d'utiliser, pour ne pas dire "sacrifier", treize de ces documents authentiques pour forger neuf pseudo-originaux pour les besoins de leur lutte contre l'évêque de Paris ${ }^{52}$. Le témoignage de Suger est comme l'écho de ce remuement archivistique d'ampleur inégalée.

\section{Le chapitre 20 (De Feins et Vendrovillare): \\ la question des »archives publiques«}

Le second passage qui me retient clôt le récit que donne Suger de ses opérations immobilières en Dunois, à Fains et Vergonville ${ }^{53}$. Suger y a racheté à Galeran de Breteuil des possessions qu'une tradition rapportée par Suger considérait comme dionysiennes par donation d'un certain Hubert de SaintGaury. Suger affecte les possessions récupérées à l'aumônerie du monastère ${ }^{54}$ et fait confirmer la transaction par un acte royal. Voici le texte de Suger ${ }^{55}$ :

Aliam etiam possessionem quae dicitur Feins et Vendrovillare, cum aliis pertinentibus villis, a Galeranno de Bretoilo et uxore ejus Juditha, et strenuo viro filio ejus Ebrardo ... multo sumptu ... comparatam, sive restitutam ... eleemosinae beati Dyonisii contulimus, sperantes de Dei misericordia quod ea pauperibus at[t]ributa eleemosina divinae retributionis beneficium

s0 Ibid. p. 262.

${ }^{51}$ Hartmut ATSMA, Jean VEZIN, Les faux sur papyrus de l'abbaye de Saint-Denis, dans: Jean KERHERVÉ, Albert RIGAUDIĖRE (dir.), Finances, pouvoirs et mémoire: mélanges offerts à Jean Favier, Paris 1999, p. 673-699, notamment p. 676.

${ }^{52}$ ATSMA, VEZIN (voir n. 51). De la précieuse liste des papyrus conservés donnée dans cet article (p. 687-690), il ressort que subsistent aujourd'hui 9 documents faux sur papyrus (pap. $\mathrm{n}^{\circ} 3 \mathrm{~b}, 4 \mathrm{~b}, 7 \mathrm{~b}, 9 \mathrm{~b}, 12 \mathrm{~b}+20 \mathrm{~b}, 13 \mathrm{~b}, 14 \mathrm{~b}, 15 \mathrm{~b}, 19 \mathrm{~b}$ ), à savoir 6 bulles pontificales, $1 \mathrm{di}-$ plôme de Dagobert $I^{\text {er }}, 1$ privilège épiscopal mérovingien, 1 procès-verbal du synode de Soissons de 862; ces 9 faux, inscrits au verso de papyrus anciens collés recto contre recto, ont nécessité la matière première d'au moins 13 documents authentiques ( 7 préceptes et 4 actes privés mérovingiens, 1 bulle du pape Formose, 1 lettre du futur abbé Maginarius). D'autres faux de la même campagne n'existent plus à l'état d'original.

${ }^{53}$ Fains-en-Dunois, Eure-et-Loir, arr. Chartres, cant. Voves; Vergonville: même localisation.

${ }^{54}$ Sur l'importance du service de l'aumônerie à Saint-Denis avant Suger, notamment sous son prédécesseur Adam, voir GROSSE (voir n. 5), p. 157-163.

55 Suger, Gesta Suggerii abbatis, éd. GASPARRI (voir n. 1), p. 88-90. 
nobis ab omnipotenti Deo misericorditer impetrabit ... Et ut in sempiternum necessitatibus pauperum firmius deserviat, precepto regis Ludovici, quod in archivis publicis repositum continetur, firmari fecimus.

Donnons les dernières lignes de l'extrait dans la traduction de Françoise Gasparri $^{56}$ :

„Et pour que [cette aumône] serve plus sûrement et pour toujours les besoins des pauvres, nous l'avons fait confirmer par un précepte du roi Louis conservé dans les archives publiques.«

Le problème n'est pas ici de traduction, mais de compréhension du texte. Que sont les »archives publiques« ici en cause? S'agit-il du chartrier du monastère ou des archives d'une autre institution? Quelles connotations renferme l'adjectif »publiques«? D'où vient cette expression?

\section{Enquête sur l'emploi de l'expression »archives publiques"}

Dans quel chartrier le diplôme délivré par Louis VI à Saint-Denis était-il conservé? Le texte de Suger, tel qu'il est transmis, ne donne aucun complément à l'expression in archivis publicis, ce qui peut débrider l'imagination ${ }^{57}$. Dans un article récemment publié dans la revue »Scriptorium«, Madame Gasparri a laissé entendre que les »archives publiques« évoquées n'étaient autres que les »archives royales $\aleph^{58}$, ce qui ferait de notre extrait un jalon capital pour l'histoire du Trésor des chartes de la monarchie capétienne. Une telle hypothèse a de quoi séduire, mais remet en cause, sans crier gare, une loi d'airain de l'archivistique altomédiévale, à savoir la conservation par le bénéficiaire des diplômes émis par le pouvoir central ${ }^{59}$. On doit par conséquent explorer la formule »archives publiques«.

56 Ibid. p. 91.

57 À cela s'ajoute le fait que le diplôme est perdu. Cf. DUFOUR, Recueil (voir n. 16), t. 2, $\mathrm{n}^{\circ} 300$ [1122-1131], p. 146-147 (édition du passage de Suger); l'Appendice II du même volume ( $\mathrm{n}^{\circ} 18$, p. 472$)$ renferme l'édition de l'acte de Galeran de Breteuil témoignant de l'acquisition rapportée par Suger, un acte dont l'abbé ne dit pas un mot!

${ }^{58}$ Françoise GASPARRI, Bibliothèques et archives de l'abbaye de Saint-Victor de Paris au XII ${ }^{e}$ siècle, dans: Scriptorium 55 (2001), p. 275-284, à la p. 281: »Mais on ne sait toujours rien sur l'organisation des archives royales, jusqu'au XII ${ }^{e}$ siècle, quand Suger dit, à propos d'une donation royale à Saint-Denis, que si quelqu'un veut vérifier ses dires, il n'a qu'à aller consulter le document qui est déposé dans les rarchives publiquesı.« Le passage auquel F. Gasparri fait allusion est bien celui qui retient notre attention, mais l'auteur extrapole quant à sa teneur réelle: Suger ne demande pas de vérifier.

${ }^{59}$ Dans l'article cité note précédente, F. Gasparri ne développe d'ailleurs pas son idée mais la prolonge de considérations portant non plus sur la conservation des actes, mais sur leur confection. 
L'expression est répandue chez les auteurs chrétiens de l'Antiquité tardive; on la rencontre chez saint Augustin $^{60}$ et ses contemporains ${ }^{61}$, ainsi que dans la traduction d'Eusèbe de Césarée par Rufin ${ }^{62}$. Les archives dont il est question désignent les documents eux-mêmes ou les dépôts qui les accueillent; il s'agit des "archives« du pouvoir impérial ou des églises, ou bien encore d'»archives « de cités ${ }^{63}$.

L'expression est passée dans le monde franc. Au singulier (in archivo publi$c o$ ), elle est attestée sous Louis le Pieux dans le proemium aux capitulaires de 818-819; l'expression renvoie assurément aux archives du souverain ${ }^{64}$. Plus anciennement et au pluriel, l'expression, sous la forme in archipibus publicis, est employée dans le formulaire mérovingien de Marculf pour désigner les gesta municipalia ayant pour fonction d'assurer la mémoire (et la validité?) des actes juridiques que les mandataires des donateurs et testateurs voulaient voir pourvus d'une firmatio publique ${ }^{65}$. L'expression in archivis publicis

${ }^{60}$ Epistola $n^{\circ} 43$, IX, 25, éd. Alois GoldBACHER, S. Aurelii Augustini opera, sectio II, Prague, Vienne, Leipzig 1898 (Corpus scriptorum ecclesiasticorum Latinorum, 34/2), p. 107, 1. 9; Epistola $\mathrm{n}^{\circ}$ 129, 4, ibid., Vienne, Leipzig 1904 (CSEL, 44), p. 37, 1. 2; Contra partem Donati post Gesta, XVI, 20, éd. Michael PETsChenIG, S. Aurelii Augustini opera, sectio II/3, Vienne, Leipzig 1910 (CSEL, 53), p. 119, I. 4; Contra Cresconium, I. III, XLV, ibid., sectio II/2, Vienne, Leipzig 1909 (CSEL, 52), p. 455; Sermones novissimi, sermo 2D (= 359B), Sermon Mayence 55, éd. François DolBEAU, Augustin d'Hippone, Vingt-six sermons au peuple d'Afrique, Paris 1996 (Collection des Études augustiniennes - Série Antiquité, 147), par. 22, p. 343, 1. 485.

${ }^{61}$ Voir les trois occurrences d'marchives publiques« dispersées dans ces documents, prodigieux notamment pour qui s'intéresse à la genèse concrète des procès-verbaux d'assemblées, que sont les Gesta collationis Carthaginensis de 411 , éd. et trad. Serge LANCEL, Actes de la conférence de Carthage en 411, 4 vol., Paris 1972-1991 (Sources chrétiennes, 194, 195, 224, 373), I, 18 (éd. cit., t. 2, p. 610-611), III, 164 (t. 3, p. 1114 1115 ) et III, 220 (t. 3, p. 1160-1161). Dans le demier cas, l'allusion vise précisément les archives du proconsul d'Afrique.

${ }^{62}$ Historia ecclesiastica, trad. Rufin, livre I, chap. 13, § 5; livre V, chap. 18, §9, éd. Theodor MOMMSEN, Eusebius Werke, t. 2/2: Die Kirchengeschichte, Leipzig 1908 (Die griechischen christlichen Schriftsteller der ersten drei Jahrhunderte, 9), p. 85, 1.16 et p. 477, 1. 22. La première citation est reprise par la Chronique de Guillaume de Tyr [† 1186], 1. 16, chap. 5, éd. Robert Burchard Constantijn HUYGENS, Turnhout 1986 (Corpus christianorum, Continuatio mediaevalis, 63), p. 721, 1. 43.

${ }^{63}$ C'est le cas de Rufin (livre I, chap. 13, 5 [voir n. 52], p. 85), à propos des warchives publiques« d'Édesse et d'Éphèse.

${ }^{64}$ Hludowici proemium generale ad capitularia tam ecclesiastica quam mundana, éd. Alfred BORETIUS, Capitularia regum Francorum, t. 1, Hanovre 1883 (MGH, Legum sectio II), $\mathrm{n}^{\circ} 137$, p. $275,1.12-15:$... libuit nobis ea quae congesta sunt ob memorie firmitatisque gratiam in unum strictim congerere et subiectis capitulis adnotare et in publico archivo recondere, ut successores Deo dispensante nostri nostra pia facta conservantes ipsi nihilominus bona facta sua successoribus suis servanda perdoceant.

${ }^{65}$ Marculfi formulae, éd. Karl ZEUMER, Formulae merowingici et karolini aevi, Hanovre 1886 (MGH, Legum sectio V), II, 38, p. 98, 1. 17-20: Et quia epistola donationis... et mandatum in te conscribtum per ordinem conditas... manifesta esse cognovimus, dignum est, ut gesta ex hoc conscribta adque subscribta tibi tradatur, et ut in archipibus publicis memo- 
s'applique tout aussi bien à des archives d'institutions ecclésiastiques. J'ai repéré trois occurrences intéressantes, dont l'origine géographique est assez proche de Suger.

La première se trouve dans un privilège intitulé au nom du pape Eugène II (824-827), un faux que les moines de Saint-Médard de Soissons ont vraisemblablement fabriqué à la fin du $\mathrm{XI}^{\mathrm{e}}$ siècle ou au début du siècle suivant ${ }^{66}$. Cette fausse bulle évoque le dépôt, »dans les archives publiques de Sainte-Marie et de Saint-Gervais « (la cathédrale de la cité), d'eulogiae accordées antérieurement par les papes Jean (Jean III, 560-573) et Grégoire (Grégoire le Grand). Ces »cadeaux « (eulogiae), dans l'esprit du faussaire tout au moins, étaient des actes pontificaux ${ }^{67}$ et c'est pourquoi lui ou d'autres, ses contemporains, $\mathrm{s}^{\prime}$ empressèrent de ressusciter ces pièces ${ }^{68}$. Voici l'extrait de la fausse bulle d'Eugène II:

Inde vir apostolicus Bandaridus Suessorum pontifex et venerabilis Ansaricus praesul eulogias ab sanctis Joanne et bonae memoriae Gregorio episcopis susceperunt, quas in archivis publicis Sanctae Mariae et Sancti Gervasii reposuerunt.

Il n'est pas du tout impossible que les faussaires aient tiré l'information - et peut-être aussi la formule - d'une source ancienne, qui pourrait dater du $\mathrm{IX}^{\mathrm{e}}$ siècle $^{69}$. On ne sait pas si le dépôt des originaux évoqués s'est fait au titre d'archives de la cathédrale, ou bien au titre des archives publiques de la cité, en somme d'archives héritières des gesta municipalia de la haute époque franque. En tout cas, l'expression in archivis publicis n'aurait rien d'anachronique, au $\mathrm{IX}^{\mathfrak{e}}$ siècle, pour désigner un dépôt ecclésiastique. L'exemple suivant va nous en convaincre.

randa servetur. Cette phrase est prononcée par le defensor et curialis, au terme de la procédure par laquelle le mandataire du donateur demande au nom de ce dernier que l'acte juridique soit validé (firmetur) et inséré (inseratur) dans les gesta publica.

${ }^{66}$ JAFFÉ-LOEWENFELD $\mathrm{n}^{\circ}$ 2562; éd. Johannes RAMACKERS, Papsturkunden in Frankreich, Neue Folge, t. 4: Picardie, Göttingen 1942 (Abhandlungen der Akademie der Wissenschaften in Göttingen, philologisch-historische Klasse, 3. Folge, 27), $n^{\circ} 2$, p. 56-62, à la p. 57. Sur cette bulle et les privilèges (faux également) qu'elle évoque, voir aussi abbé Michel DELANCHY [ $\dagger$ 1979], Étude historique [ouvrage posthume], dans: Denis DEFENTE (dir.), Saint-Médard. Trésors d'une abbaye royale, Paris 1996, p. 101-102 et 116.

${ }^{67}$ On rapprochera ces eulogiae du munus rapporté de Rome par l'évêque de Trèves (voir la n. 41).

${ }^{68}$ Jean III: JAFFÉ-KALTENBRUNNER $\mathrm{n}^{\circ} 1039$ (11 mars 562). Grégoire $\mathrm{I}^{\mathrm{er}}$ : JAFFÉ-EWALD $n^{\circ} 1239$ (25 mai 593). Des documents intitulés au nom de ces deux papes existent encore, mais il s'agit de faux, »de la même veine et très probablement du même atelier« que le faux privilège d'Eugène II, si on en croit DELANCHY (voir n. 66), p. 102. Le même érudit croit pouvoir reconnaître dans le texte de la fausse bulle d'Eugène II des bribes »qui auraient pu appartenir à [un] acte authentique« de ce pape. L'hypothèse, sensée a priori, devrait être validée par une étude précise du dossier, qui manque encore.

${ }^{69}$ Voir la note précédente. 
Il s'agit de l'Historia translationis sancti Benedicti, composée par le moine de Fleury Adrevald dans la deuxième moitié du $\mathrm{IX}^{\mathfrak{e}}$ siècle $^{70}$. Au début de l'ouvrage, Adrevald retrace les débuts de son monastère et évoque un acte (aujourd'hui perdu), par lequel le roi, sans doute Clovis II, avait cédé à Leodebaudus son domaine de Fleury en échange d'Attigny, domaine qui deviendra le palais bien connu ${ }^{71}$. Adrevald signale alors que ce testamentum fondamental "est encore conservé dans les archives publiques de notre monastère«.

Igitur ab utrisque partibus mutuae commutationes factae sunt: memorato abbate dante quod a genitoribus ei relictum est praedium; et rege supradicto, reciproca vicissitudine, pro eo, agellum quemdam Floriacum cognominatum cum appendiciis, haud longe a littore Ligeris. Quod testamentum mutuae vicissitudinis usque hodie in archivis publicis nostri reservatur monasterii ${ }^{72}$.

Le dernier témoin à citer date du milieu du $\mathrm{XI}^{\mathrm{e}}$ siècle et provient du monastère de Saint-Bertin. Dans la Relatio de inventione et elevatione S. Bertini qu'il écrit peu après 1052, l'abbé Bovon (1042-1065) transcrit, entre autres documents, la lettre qu'avait adressée l'évêque de Thérouanne Drogon (10301078) à l'archevêque de Reims Gui, au sujet de l'inventio des reliques de saint Bertin $^{73}$. Précisons dès maintenant - ce n'est pas indifférent au développement qui suivra - que cette lettre de Drogon a été probablement rédigée avec le concours des bertiniens ou par Bovon lui-même, et donc qu'elle reflète le bagage littéraire d'un moine de Saint-Bertin. »On lit«, écrit Drogon, »dans les archives publiques de ce monastère « que saint Folquin, évêque de Thérouanne $(\dagger 855)$, avait caché en terre le corps de saint Bertin peu de temps avant les attaques normandes.

${ }^{70}$ Adrevald, actif jusque vers $878 / 9$ a dû rédiger l'Historia translationis après 865 . Voir Alexandre VIDIER, L'historiographie à Saint-Benoît-sur-Loire et les »Miracles de saint Benoît«, ouvrage posthume revu et annoté par les soins des moines de l'abbaye de SaintBenoit de Fleury, Paris 1965, p. 157-158; dom Jacques HOURLIER, La translation d'après les sources narratives, dans: Le culte et les reliques de saint Benoit et de sainte Scholastique (= Studia monastica 21 [1979]), p. 213-239.

7 Sur les débuts de Fleury, voir notamment: dom Jean LAPORTE, Vues sur l'histoire de l'abbaye de Fleury aux VII $^{e}$ et VIII ${ }^{e}$ siècles, dans: Le culte (voir n. 70), p. 109-143. Josiane BARBIER a entrepris un réexamen de fond du dossier diplomatique de Leodebaudus; voir notamment son étude: La reine fait le roi. Une révision de la datation du testament de Leodebaudus, à paraître dans les Mélanges en l'honneur de Michel Parisse.

${ }^{72}$ Chap. II, éd. Eugène DE CERTAIN, Les Miracles de Saint-Benoît, Paris 1858 (Société de l'histoire de France), p. 2-3.

${ }^{73}$ Bibliotheca hagiographica latina [BHL] n ${ }^{\circ}$ 1296; éd. Oswald HOLDER-EGGER, dans: MGH, Scriptores, t. 15/1, p. 524-534. Sur l'œuvre, voir Karine UGÉ, Relics as tools of power: the eleventh-century Inventio of St-Bertin's relics and the assertion of Abbot Bovo's authority, dans: Arnoud-Jan Busterveld, Henk TheUNIS, Andrew WareHAM (dir.), Negotiating secular and ecclesiastical power. Western Europe in the central Middle Ages, Turnhout 1999 (International medieval research, 6), p. 51-71. 
Legitur in ipsius loci archivis publicis, quod. S. Folquinus nostrae sedis episcopus (cujus etiam corpus magnis celebre virtutibus in eadem quiescit ecclesia) grassante igne et ferro Danorum barbarie per loca maritima, paulo ante hanc vastationis luem corpus hujus sancti certa die terra recondiderit ... ${ }^{74}$.

Les exemples d'Adrevald et de Bovon montrent qu'au $\mathrm{IX}^{\mathrm{e}}$ comme au $\mathrm{XI}^{\mathrm{e}}$ siècle, les archives d'un monastère étaient parfois qualifiées d'»archives publiques « par les moines du lieu ou par des tiers très proches de ces moines. N'en serait-il pas de même sous la plume de Suger? C'est d'autant plus vraisemblable qu'il est impensable que le diplôme de Louis VI sollicité par Suger se soit trouvé conservé de façon habituelle (on notera le terme repositum!) hors du chartrier de la communauté de Saint-Denis. Si Suger avait demandé au roi un diplôme destiné à garantir les possessions nouvellement acquises de Fains et de Vergonville, et leur affectation à l'aumônerie, c'était naturellement pour avoir le précieux précepte auprès de lui. D'ailleurs, on a déjà dit plus haut que c'est la destination naturelle de toute charte originale, et spécialement de tout diplôme royal, que d'être conservée dans les archives du bénéficiaire ${ }^{75}$. On

${ }^{74}$ Éd. HOLDER-EGGER (voir n. 73), p. 531, 1. 10.

${ }^{75}$ Au-delà de la question générale des traces qu'à l'époque de Suger une »chancellerie«, qu'elle fût royale, princière ou épiscopale, pouvait conserver des actes qu'elle expédiait, on s'est demandé, en présence d'actes en double expédition ou sous forme de chirographe, si parfois l'un des exemplaires n'était pas destinée à gagner les archives de l'expéditeur, non pas à titre de co-contractant, mais à titre d'autorité émettrice. Au début du XII ${ }^{e}$ siècle, certains chirographes délivrés par la chancellerie épiscopale de Noyon-Tournai semblent en effet relever de cette mission: Laurent MORELLE, Archives épiscopales et formulaire de chancellerie au XII ${ }^{e}$ siècle. Remarques sur les privilèges épiscopaux connus par le Codex de Lambert de Guînes, évêque d'Arras (1093/94-1115), dans: Christoph HAIDACHER, Werner KOFLER (dir.), Die Diplomatik der Bischofsurkunde vor 1250/La diplomatique épiscopale avant 1250. Referate zum VIII. Internationalen KongreB für Diplomatik, Innsbruck, 27. September-3. Oktober 1993, Innsbruck 1995, p. 255-267, aux p. 255-256; cf. Brigitte BEDOS-REZAK, Towards an Archeology of the Medieval Charter: Textual Production and Reproduction in Northern French 'Chartriers', dans: Charters (voir n. 6), p. 43-60; au IX' siècle, l'établissement d'actes en deux exemplaires pour raison d'archivage semble attesté: voir Irmgard FEES, Drei Urkunden des Bischofs Jonas von Autun und die (angebliche) Synode von Saints-Geosmes, dans: Deutsches Archiv 51 (1995), p. 375-404, aux p. 383 et ss. Pour les actes de Louis VI, la pratique, rare, du chirographe ne semble rien devoir à des préoccupations d'archivage, pas plus que celle des doubles ampliations: voir les références dans DUFOUR, Recueil (voir n. 16), t. 3: Introduction, p. 70-71; les présomptions de GASPARRI (voir n. 58), p. 283 sont vagues. - Pour les archives princières, on relèvera le cas flamand suivant: dans une charte de grande portée du 8 février $110[0]$ par laquelle il soumet Saint-Bertin à Cluny (éd. Fernand VERCAUTEREN, Actes des comtes de Flandre [10711128], Bruxelles 1938 [Commission royale d'histoire, Recueil des actes des princes belges, 2], $\mathrm{n}^{\circ} 34, \mathrm{p} .100$, à lire avec les corrections de Joseph-M. DE SMET, Quand Robert II confiat-il Saint-Bertin à Cluny?, dans: Revue d'histoire ecclésiastique 46 [1951], p. 160-164), le comte de Flandre Robert II déclare mavoir conservé entre ses mains un exemplaire de cette charte (Porro, ut haec nostra concessio immo supplicatio imperpetuum rata atque illibata 
objectera peut-être qu'il manque un complément à l'expression, là où Adrevald et Bovon/Drogon (sans parler du faussaire de Saint-Médard) parlent des »archives publiques« »du lieu« ou »de notre monastère«. L'argument ne me semble pas décisif. On peut penser en effet que pour Suger, les sarchives publiques« renvoyaient de façon si évidente au chartrier dionysien qu'une précision s'avérait inutile. Le public du De administratione était formé au premier chef de la communauté des dionysiens ${ }^{76}$; celle-ci savait à quoi s'en tenir. Le contexte est à cet égard très différent de l'Historia translationis d'Adrevald et de la lettre de Drogon de Thérouanne. À bien y réfléchir d'ailleurs, l'absence de tout déterminatif surprendrait davantage si l'expression "archives publiques« visait les archives royales, car devant une telle anomalie archivistique, Suger aurait sans doute jugé utile d'apporter une précision permettant aux lecteurs et auditeurs de ne pas se méprendre. Comme Suger ne l'a pas fait, on peut croire que le texte se donnait à comprendre selon une interprétation finalement banale.

Concluons et résumons: les »archives publiques« où reposait le diplôme de Louis VI ne sont rien d'autre que le chartrier du monastère. Comme dans le cas de biblos étudié plus haut, acceptons de déchanter: Suger ne nous fait pas découvrir une réalité cachée et révolutionnaire.

\section{Les résonances de l'expression »archives publiques«}

Cette mise au point faite, on peut se demander d'où vient, chez Suger, l'expression warchives publiques«. On peut songer à saint Augustin, mais les »archives publiques« évoquées par le Père de l'Église ne sont pas celles d'un monastère. Une nouvelle fois, on est tenté de regarder du côté de l'œuvre d'Adrevald de Fleury. Et curieusement, cette piste vaut aussi bien pour la lettre rapportée par l'abbé Bovon de Saint-Bertin que pour le texte rédigé par l'abbé Suger. À Saint-Bertin en effet, on disposait de l'œuvre d'Adrevald dès le début du $\mathrm{XI}^{\mathrm{e}}$ siècle $^{77}$ et Karine Ugé a d'ailleurs estimé que celle-ci avait

permaneat, carte hujus exemplar penes nos retinere decrevimus). Même dans une affaire politique capitale, on voit que ce n'est pas l'original, mais une copie (exemplar), qui est conservée dans les dossiers comtaux; l'original est allé à Cluny.

${ }^{76}$ Voir le prologue du De administratione, éd. GASPARRI (voir n. 1), p. 54; cf. Lindy GRANT, Abbot Suger of Saint-Denis: Church and State in Early Twelfth-Century France, Londres, New York 1998 (The Medieval World), p. 34 et 207.

${ }^{77}$ Il s'agit du ms. Bibl. mun. Saint-Omer 350, copié à l'époque de l'abbé Odbert (986-1007); voir André WILMART, Les livres de l'abbé Odbert, dans: Bulletin de la Société des antiquajres de la Morinie 14 (1922-29), p. 169-188, aux p. 178-179. S'agissant des cuvres d'Adrevald, le manuscrit juxtapose l'Historia translationis $S$. Benedicti (BHL $\mathrm{n}^{\circ} 1117$ ) et des extraits du premier livre des Miracula, selon des modalités qui caractérisent une branche de la tradition des œuvres en cause bien identifiée par VIDIER (voir n. 70), p. 170, § c. 
inspiré la composition de la Relatio $^{78}$ : l'expression in archivis publicis vient confirmer l'hypothèse. Quant à la bibliothèque de Saint-Denis, elle conservait aussi une copie de l'Historia translationis, qui formait la troisième section d'un légendier composite qui en comportait quatre, écrites entre le début du $\mathrm{X}^{\mathrm{e}}$ siècle et le $\mathrm{XI}^{\mathrm{e}}$ siècle; la table liminaire du codex, écrite fin $\mathrm{XI}^{\mathrm{e}}$ ou début $\mathrm{XII}^{\mathrm{e}}$ siècle, atteste que le manuscrit était déjà assemblé à l'époque de Suger ${ }^{79}$. Qui plus est, d'après cette table, l'Historia translationis faisait l'objet d'une lecture le 10 juillet, pour la fête de la translation de saint Benoît. Si, comme c'est probable, le manuscrit se trouvait déjà entre les mains des moines dionysiens à l'époque de Suger, on peut imaginer que c'est à l'occasion d'une de ces lectures rituelles que l'expression in archivis publicis a été entendue et assimilée par Suger. Le manuscrit de la Bibliothèque vaticane pourrait être le maillon joignant l'œuvre fleurisienne au De administratione dionysien.

Il reste à se demander de quelle signification particulière était chargé l'adjectif "public«, en quoi l'expression in archivis publicis pouvait séduire un lecteur au point d'entrer dans son bagage lexicographique. Plusieurs réponses sont envisageables. Adrevald comme Suger emploient le terme à propos d'actes royaux: publicus peut donc renvoyer à la nature des actes conservés. On trouve cette acception dans un passage de la Vita ancienne de l'abbé réformateur Poppon de Stavelot ( $\dagger$ 1048), rédigée peu après 1058, où l'expression »archives publiques « désigne les chartes délivrées par le marquis de Flandre Baudouin IV, dont l'autorité princière est assurément »régalienne ${ }^{80}$. Si l'on adopte cette manière de voir, on pourrait même concevoir que les »archives publiques« de Suger désignaient alors la section du chartrier où l'on serrait les actes d'autorité, les actes qu'on appelle "publics«.

L'hypothèse ne s'applique pas cependant au cas de Saint-Bertin. Le texte qu'on pouvait lire »dans les archives publiques« du monastère flamand n'était pas un acte royal, pas même un document d'archives, mais une simple narra-

${ }^{78}$ UGÉ (voir n. 73), p. 63; d'après l'auteur, l'insertion des lettres échangées entre Drogon de Thérouanne et Gui de Reims dans la Relatio serait un procédé inspiré de l'insertion de la lettre du pape Zacharie dans les Miracula (I, chap. XV, éd. DE CERTAIN [voir n. 72], p. 3839); sur cette lettre, voir Jacques HouRLIER, La lettre de Zacharie [avec un appendice de J. Laporte], dans: Le culte (voir n. 70), p. 241-252.

79 Il s'agit du ms. Bibl. Vat., Reg. lat. 528, fol. 185-192; voir Albert PONCELET, Catalogus codicum hagiographicorum latinorum bibliothecae Vaticanae, Bruxelles 1910 (Subsidia hagiographica, 11), p. 354-356; Thomas HEAD, Hagiography and the Cult of Saints, the Diocese of Orléans 800-1200, Cambridge 1990 (Cambridge studies in medieval life and thought, $4^{\mathrm{e}}$ série, 14), p. 127, n. 108; cf. VIDIER (voir n. 70), p. 143. Ce codex, qui porte une mention d'appartenance du XIII ${ }^{\mathrm{e}}$ siècle (hic est liber beati Dionysii), ne semble pas répertorié dans NEBBIAI-DALla GuARDA (voir n. 14).

${ }^{80}$ Vita Popponis Stabulensis auctore Everhelmo, éd. Wilhelm WATTENBACH, dans: MGH, Scriptores, t. 11, Hanovre 1854, p. 293-316, à la p. 300, 1. 22-24: nam annuente praefato marchiso res monasterii a militibus callide prudenterque repetiuit atque ut eiusdem loci fratribus permanerent publicis archiuis ratum stabiliuit. 
tion. L'enfouissement du corps de saint Bertin est en effet rapporté par le fameux chroniqueur Folcuin, dans deux œuvres différentes: d'abord dans ses fameux Gesta et traditiones de Sithiu, rédigés en 961/62 à l'époque où il était moine de Saint-Bertin, puis dans sa Vita Folquini composée après qu'il fut devenu abbé de Lobbes en $965^{81}$. Dans le cas de Saint-Bertin, les »archives publiques« correspondraient donc à un ensemble assez mou de références officielles et "autorisées«, un statut auquel l'œuvre historiographique de Folcuin a d'ailleurs bien vite accédé ${ }^{82}$. À cela s'ajoute peut-être le fait que la personne de l'évêque étant publique et le culte des reliques ne l'étant pas moins, cette qualité rejaillissait sur la documentation hagio- ou historiographique qui le concernait, une documentation tournée vers l'extérieur.

Une troisième possibilité nous est offerte. La qualité "publique« des archives ne dépend pas seulement de la nature des pièces d'archives ("Archivalien«), elle a également à voir avec la qualité que l'on reconnaît au dépôt d'archives ("Archiv«) qui les abrite et à l'institution qui en est responsable. À cet égard, on peut dire, quitte à risquer l'anachronisme, que les communautés monastiques avaient conscience de remplir une "mission de service public ${ }^{83}$, garantie par l'autorité des rois et des princes: les chartriers monastiques n'étaient pas de simples réceptacles, mais des loca credibilia; ils constituaient en quelque sorte, et d'autres dépôts d'archives ecclésiastiques avec eux, les gesta municipalia de l'âge seigneurial. L'éclatement progressif des formulaires et la mainmise des moines et clercs sur la production de l'écrit, bien marquée à partir du $\mathrm{X}^{\mathrm{e}}$ siècle, $\mathrm{n}$ 'ont pu qu'aviver l'idée que les chartriers monastiques étaient "publics«, à l'instar de leurs biens que les moines considèrent parfois comme des res publica $e^{84}$. On comprend alors que l'expression in archivis

${ }^{81}$ Gesta abbatum Sancti Bertini Sithiensium, éd. Oswald HoLDER-EGGER, dans: MGH, Scriptores, t. 13, Hanovre 1881, chap. 58, p. 618; Vita Folquini episcopi Morinensis (BHL $\mathrm{n}^{\circ}$ 3079), éd. HOLDER-EGGER, dans: MGH, Scriptores, t. $15 / 1$, Hanovre 1887 , chap. 7 , p. 428.

82 Je me permets de renvoyer ici à mon mémoire d'habilitation intitulé «Autour de Folcuin de Saint-Bertin«, présenté en décembre 2001 (Université Paris I) et dont la publication est prévue dans la collection des Mémoires et documents de l'École des chartes.

${ }^{83}$ Il conviendrait sans doute d'explorer en profondeur l'articulation des notions de "public de »sacré«, à partir notamment de la formule du Digeste, 1, 8 (... Sanctae autem res sunt hae quae publice consecratae sunt, non private). Par un certain renversement et dans un certain contexte, la notion de public pourrait impliquer la dimension sacrée du lieu. Sur les archives comme dépôt sacré: Olivier GUYOTJEANNIN, »Penuria scriptorum«: le mythe de l'anarchie documentaire dans la France du nord ( $\mathrm{X}^{\mathrm{e}}$-première moitié du $\mathrm{XI}^{\mathrm{e}}$ siècle), dans: ID., Laurent MORELLE, Michel PARISSE (dir.), Pratiques de l'écrit documentaire au $\mathrm{XI}^{e}$ siècle (= Bibliothèque de l'École des chartes 155 [1997]), p. 11-44, à la p. 31.

${ }^{24}$ Sur ce demier point, voir l'exemple, contemporain de Suger, donné par l'Histoirepolyptyque de l'abbaye de Marchiennes (1116/1121), éd. Bernard DELMAIRE, Louvain-laNeuve 1985 (Centre belge d'histoire rurale, 84), § 2, p. 66: contre les chicaneurs de tout poil qui inquiètent les monastères à propos de l'origine de leurs biens, l'auteur vante les mérites de la connaissance bien documentée du passé: ...reor multum proficuum fore contra insur- 
publicis ait imprégné la mémoire utile de l'abbé Suger: elle s'accordait harmonieusement au "public role ${ }^{85}$ que l'abbé destinait à son monastère, un rôle que traduisait l'expression de caput regni qu'on rencontre dans un diplôme célèbre rédigé à Saint-Denis ${ }^{86}$. Mais comme Suger a simplement remployé une formule déjà en circulation, et sans aucune précision ni commentaire, rien ne nous autorise à la gloser comme si elle témoignait d'une réflexion spéculative sur la signification du chartrier dionysien dans le cadre de la mission politique dévolue à l'abbaye de Saint-Denis par son chef.

Au total, le réexamen des deux passages du De administratione conduit à un certain désenchantement. Sur le terrain archivistique - comme sur d'autres semble-t-il -, Suger n'innove pas. Il ne faut pas se laisser abuser par les mots qu'il emploie, dont on est porté à surestimer la profondeur à cause d'une tradition historiographique pesante. Le terme biblos, qui signifie "papyrus« et non pas »cartulaire«, n'invite pas à faire de Suger le témoin lucide et moderne d'un chartrier repensé dans ses composantes; l'expression warchives publiques« qu'il applique au chartrier du monastère, réminiscence possible d'une lecture d'origine fleurisienne (Adrevald de Fleury), ne suppose pas forcément un nouveau projet »politique« pour la mémoire écrite dionysienne.

Mais ce que nous avons perdu d'un côté, nous le regagnons de l'autre. On saisit par la prose de Suger la forte présence des papyrus dans l'image du chartrier dionysien et la facilité avec laquelle l'abbé assimile des notions apprises au cours de sa vie monastique. Suger gagne en consistance ce qu'il perd en brillant. Si l'on ajoute que le passage relatif à Argenteuil invite à considérer avec beaucoup de méfiance les assertions les plus nettes de Suger et peut-être aussi à mieux scruter la genèse d'un texte (le De administratione) moins lisse qu'il ne semble au premier abord, la moisson n'est finalement pas si pauvre.

gentium infestationes et adversus omnium violentiam injuste occupare querentium res $p u$ blicas.

${ }^{85}$ GRANT (voir n. 76), p. 26.

${ }^{86}$ L'expression est dans l'acte de Louis VI de 1124, éd. DUfOUR, Recueil (voir n. 16), t. 1, $n^{\circ} 220$, p. 458-466, à la p. 466 (texte) et p. 463 (singularité de la formule). 
